\title{
Acoustic Imaging of In-Duct Aeroengine Noise Sources Using Rotating Beamforming and Phased Arrays
}

\author{
Luciano C. Caldas, Member, IEEE, Paulo C. Greco Jr., Carlos C. Pagani, and Luiz A. Baccalá, Member, IEEE
}

\begin{abstract}
An in-duct rotating beamforming technique, using a wall-mounted array of microphones placed within a long duct, was developed for locating broadband noise sources from an aeroengine fan. The technique was experimentally implemented at the recently constructed long-duct low-speed fan rig test facility at the University of São Paulo in Brazil. The test rig has a 16-bladed fan rotor and 14-vaned stator, with speed up to $4250 \mathrm{r} / \mathrm{min}$ and a maximum 0.1 Mach number mean axial flow speed. Here, we describe the beamforming details with an emphasis on the mandatory coordinate change to a rotating reference frame for a frequency domain virtual rotating microphone technique. Example sound source maps are presented revealing clear noise blade signatures together with simulated point spread function maps using modal steering vectors at six different frequencies that characterize the beamformer.
\end{abstract}

Index Terms-Aeroacoustic, array signal processing, fan noise, in-duct beamforming, power spectral density, rotating acoustic imaging.

\section{INTRODUCTION}

$\mathbf{E}$ NVIRONMENTAL noise pollution is calling for quieter products worldwide.

This is a promising research line greatly benefiting from the use of scaled aircraft models [1] and wind tunnel test facilities that allow connecting experimental and numerical approaches [2]. This effort has been spearheaded by breakthrough techniques in signal and image processing as applied to acoustic field analysis. Of particular relevance here is the now ubiquitous Acoustic Beamforming, which employs a microphone array to map acoustic sources.

Manuscript received July 1, 2016; revised June 8, 2017; accepted June 13, 2017. Date of publication June 29, 2017; date of current version August 4, 2017. The USP fan rig test facility was supported by the Federal Funding Authority for Studies and Projects. The guest editor coordinating the review of this manuscript and approving it for publication was Prof. Yoav Schechner. (Corresponding author: Luciano C. Caldas.)

L. C. Caldas is with the Department of Electrical Engineering, Escola Politécnica, University of São Paulo, São Paulo 05508-010, Brazil (e-mail: lucianocaldas@usp.br).

P. C. Greco Jr. is with the Department of Aeronautical Engineering, University of São Paulo, São Carlos 13566-590, Brazil (e-mail: pgreco@sc.usp.br).

C. C. Pagani is with São Paulo State University, São Paulo 13876-750, Brazil (e-mail: carlos.pagani@sjbv.unesp.br).

L. A. Baccalá is with the Department of Telecommunications and Control Engineering, Escola Politécnica, University of São Paulo, São Paulo 05508-010, Brazil (e-mail: baccala@lcs.poli.usp.br).

Color versions of one or more of the figures in this paper are available online at http://ieeexplore.ieee.org.

Digital Object Identifier 10.1109/TCI.2017.2721744
As an illustration, measuring the sound level due to an aircraft during its landing approach from some point on the ground, bears contributions from all noise generating mechanisms: propulsion systems, landing gears and so on. Airframe noise constitutes a category of its own composed of concurrent flow-induced sound sources; among the most important ones come forming the landing gears and the high-lift system [2]-[4]. Single microphone aircraft noise measurements lead to spectral analyses of the recorded signals whose noise signature comes from the acoustic field, but fail to provide quantitative information about the contribution due to each noise generating source. Beamformers play the role of spatial filters that directionally break down the various noise sources [5]-[7] through an image map whose contour levels portray the pressure density over some preselected spatial region of interest.

What distinguishes the present work from others in the literature is its application to an unconventional environment that involves identifying broadband rotating sources due to fan spinning blades within a long-duct. Measurements within this environment have been recently carried out at some test facilities [8]-[12]. The difficulty faced in characterizing sound propagation within a duct due to rotating sources lies in the specificity of the required beamforming technique for this scenario. The aforementioned method from Dougherty et al. differs from ours not just because of different test facility characteristics, but also because it employed the (VRM) technique based on time domain interpolation, as opposed to our use of an exclusively frequency domain approach. To the best of our knowledge, this technique was only tested experimentally before by Pannert et al. [13] within a free-field context rather than in the present confined ducted environment. A comparison of these two techniques applied to ducted sources has only recently been carried out by us in [14].

After presenting the necessary associated formulation and showing how to compute the effects of rotating noise sources inside a duct via modal steering vectors (Section II), we discuss the details of reducing the beamforming to a rotating reference frame topped by in-duct point spread function map examples. Section III covers spectral estimation under the virtual rotating method while Section IV describes the experimental setup followed by example results in Section V. Section VI concludes the paper. 


\section{BEAMFORMING REVIEW}

Mathematically, beamforming is an attempt to solve the "inverse problem", i.e., whereby the observation of the magnitude of a physical quantity at a point, pressure at microphones from an array in our case, allows inferring its value at other points.

To apply it, one must first select a region of interest for mapping its acoustic sources. This region is discretized into a grid of points, each of which is related to the data measured by the microphone array through a frequency-dependent complexvalued steering vector that essentially contains the phase-delay information associated with the acoustic waves traveling from those points towards the microphone array. This requires knowing how sound waves propagate under those circumstances. For the in-duct propagation case, the propagation equation must be supplemented with adequate boundary conditions.

The beamforming estimate of the pressure density $b_{s}$ at a grid point $\xi_{s}$ is given by:

$$
b_{s}=\vec{w}_{s} \mathbf{C} \vec{w}_{s}^{H}
$$

where

$$
\overrightarrow{w_{s}}=\frac{\overrightarrow{g_{s}}}{\left\|\overrightarrow{g_{s}}\right\|}
$$

$\mathrm{C}_{N \times N}$ is the cross-spectral matrix at frequency $\omega$ relating the available array microphone pairs, (more on this in Section III-B). Vector $\overrightarrow{g_{s}}$ is called the steering vector and works as a spatial filter focusing the array at some grid point of interest by relating the phase-shift due to the wave path delay in going from $\xi_{s}$ to each microphone position $\vec{x}_{i}$. One may explicitly write

$$
\begin{aligned}
\overrightarrow{g_{s}} & =\vec{g}\left(\vec{x}, \vec{x}_{s}, \omega\right)=\ldots \\
& =\left[g_{1}\left(\vec{x}_{1}, \vec{x}_{s}, \omega\right), g_{2}\left(\vec{x}_{2}, \vec{x}_{s}, \omega\right), \ldots, g_{N}\left(\vec{x}_{N}, \vec{x}_{s}, \omega\right)\right]
\end{aligned}
$$

where each element $g_{i}\left(\vec{x}_{i}, \vec{x}_{s}, \omega\right)$ represents the transfer function associated with the wave path linking microphone $\overrightarrow{x_{i}}$ to grid point $\xi_{s}$ at frequency $\omega$. To define $g_{i}\left(\vec{x}_{i}, \vec{x}_{s}, \omega\right)$, one must solve the convected wave equation under the appropriate boundary conditions

$$
\left(\nabla^{2}-\frac{1}{c^{2}} \frac{D^{2}}{D t^{2}}\right) p(t, \vec{x})=0
$$

where $c$ is the sound speed in a uniform flow and $M$ the Mach number. $D / D t=\partial / \partial t+c M(\partial / \partial z)$ is the derivative operator associated with a mean flow velocity of $c M$ in the $z$ direction (axial direction for a duct). The adopted coordinate conventions are shown in Fig. 1. To deal with in-duct wave propagation, 4 must be supplemented with hard-wall duct conditions, discussed next.

\section{A. Modal Steering Vector}

In-duct sound propagation can be described as a superposition of "modes" where each mode is an eigen-solution of the wave equation that takes duct boundary conditions into account. The appropriate hard-wall circular duct condition for our case is expressed by

$$
\left.\frac{\partial p}{\partial r}\right|_{r=a}=0
$$

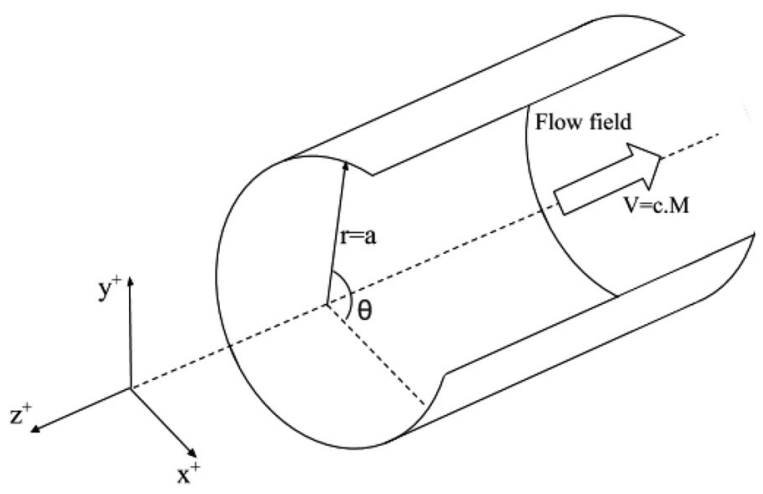

Fig. 1. Coordinate system convention.

where $a$ is the duct radius.

Hence the solution of 4 is given by

$$
\begin{aligned}
p(\omega, \vec{r})= & \sum_{m=-\infty}^{\infty} \sum_{n=0}^{\infty} A_{m, n}^{+} \Phi_{m, n}^{+}(r, \theta) e^{-j k_{z}^{+} z}+\ldots \\
& +\sum_{m=-\infty}^{\infty} \sum_{n=0}^{\infty} A_{m, n}^{-} \Phi_{m, n}^{-}(r, \theta) e^{-j k_{z}^{-} z}
\end{aligned}
$$

where superscripts ${ }^{+}$and ${ }^{-}$indicate variables associated with positive and negative z-direction propagation, following Fig. 1 conventions.

The acoustic modal functions $\Phi_{m, n}(r, \theta)$ can be written as

$$
\begin{array}{r}
\Phi_{m, n}(r, \theta)=\frac{J_{m}\left(k_{m, n} r\right)}{N_{m, n}} e^{j m \theta}, \quad m=0, \pm 1, \pm 2, \ldots \\
n=0,+1,+2, \ldots
\end{array}
$$

where $J_{m}(\cdot)$ stand for Bessel functions of the first kind, $N_{m, n}$ is a mode normalization constant discussed in Appendix A and $k_{m, n}$ is the mode eigenvalue. The solution in 7 is completely defined by finding the correct eigenvalue $k_{m, n}$ from the characteristic equation that satisfies the hard-wall boundary condition in 5 . These values must satisfy $J_{m}^{\prime}\left(k_{m, n} a\right)=0$, i.e., the product $k_{m, n} a$ is the $n$th stationary point of $J_{m}(x)$, see [15] for a table of these values. Hence for these $k_{m, n} a$ stationary values, there is a combination of modes $(m, n)$ associated with the axial wave number $k_{z}^{(-)}$:

$$
k_{z}^{(-)}=\frac{-k_{0} M-\sqrt{k_{0}^{2}-\left(1-M^{2}\right) k_{m, n}^{2}}}{1-M^{2}}
$$

where $\mathrm{M}$ is the Mach number and $k_{0}$ is the free wave number $k_{0}=w / c$.

The formulation for modal steering vectors relies on Green's function solutions of the wave propagation within a duct ([16], [17]) which, in turn, corresponds to the steering vectors suggested by Dougherty et al. [9]:

$$
\begin{aligned}
g\left(\overrightarrow{r_{i}}, \overrightarrow{r_{s}}, \omega\right)= & \sum_{m=-m^{-}}^{m^{+}} \sum_{n=0}^{n^{+}} \frac{J_{m}\left(k_{m, n} r_{i}\right) J_{m}\left(k_{m, n} r_{s}\right)}{N_{m, n}^{2}} \\
& \times \ldots e^{j m\left(\theta_{i}-\theta_{s}\right)} e^{-j k_{z}^{(-)}\left(z_{i}-z_{s}\right)}
\end{aligned}
$$


where subscripts $i$ and $s$ refer to microphone and grid point indexes, respectively. $k_{z}^{(-)}$is the axial wave number with ${ }^{(-)}$ standing for upstream acoustic propagation from the fan towards the microphone array.

The $m^{-} \leq m \leq m^{+}$and $0 \leq n \leq n^{+}$terms in 9 follow from 10 and list the cut-on modes. A given mode $(m, n)$ is called cut-on when 8 is a strictly real number

$$
k_{0}^{2}-\left(1-M^{2}\right) k_{m, n}^{2}>0
$$

or equivalently

$$
k_{m, n}<\frac{k_{0}}{\sqrt{1-M^{2}}}
$$

Otherwise it is a cut-off mode that decays exponentially along the z-direction. In the present work, only cut-on modes are used.

\section{B. Rotating Reference Frame}

The above formulation holds only for the in-duct beamforming of stationary sources. If sources are rotating, as is the case of sources associated with fan blades, one must adapt the formulation to a rotating reference frame, as detailed by Rienstra and Hirschberg [16], Dougherty [9] and Lowis [12]. This may be accomplished by the transformation:

$$
(r, \theta, z, t) \quad \rightarrow \quad\left(r^{\prime}, \theta^{\prime}, z^{\prime}, t^{\prime}\right)
$$

where

$$
r^{\prime}=r, \quad \theta^{\prime}=\theta-\Omega t, \quad z^{\prime}=z \quad t^{\prime}=t
$$

where $\Omega$ is the frame angular rotation speed. Substituting the latter into (4) leads to

$$
\begin{aligned}
k_{0}^{\prime} & =k_{0}-\frac{m \Omega}{c} \\
\omega^{\prime} & =\omega-m \Omega \\
k_{z}^{(-)} & =\frac{-k_{0}^{\prime} M-\sqrt{{k_{0}^{\prime}}^{2}-\left(1-M^{2}\right) k_{m, n}^{2}}}{1-M^{2}}
\end{aligned}
$$

where the primed quantities refer to the new rotating reference frame. (11b) expresses the frequency-shift caused onto the spinning modes generated by rotating sources. Signal frequencies in rotating frame $\omega^{\prime}$ correspond to $\omega$ ones in the static frame. Then, substituting (11) into (9) leads to the rotating frame steering vector:

$$
\begin{aligned}
g\left(\overrightarrow{r_{i}}, \overrightarrow{r_{s}}, \omega^{\prime}\right)= & \sum_{m=-m^{-}}^{m^{+}} \sum_{n=0}^{n^{+}} \frac{J_{m}\left(k_{m, n} r_{i}\right) J_{m}\left(k_{m, n} r_{s}\right)}{N_{m, n}^{2}} \\
& \times \ldots e^{j m\left(\theta_{i}-\theta_{s}\right)} e^{-j k_{z}^{\prime(-)}\left(z_{i}-z_{s}\right)}
\end{aligned}
$$

The present formulation allows computing the rotating frame steering vector. It is formally similar to (9) except for a frequency shift to $\omega$ and its allied effect on the axial wave number $k_{z}^{(-)}$. Since beamforming imaging via (1) requires computing the (CSM), one also needs its value in the rotating frame. Section III-C describes this in detail.

\section{Array Point Spread Function - PSF}

The (PSF) describes how an imaging system responds to a point source input. Ideally, a point source should correspond to a single pixel in the beamforming image. However, due to the array's geometry (length, distance from the source, microphone relative placement and the signal frequency of interest) the final image spreads over many image pixels. Point-spread functions portray this spread and represent an imaging index of merit that is commonly used to measure the array's performance in terms of beam-width (BW) and side-lobe level (or dynamic range DR) rejection: the narrower the beam and the lower the side lobe levels, the better the array imaging performance.

To represent the PSF map, a point source must be modeled in terms of its radiation directivity depending on the type of source under study. Here, sources are treated as dipoles following Lowis [12] and Sitjsma [18]. The effect of a dipole stationary tonal source, denoted $\vec{j}$, was simulated following the guidelines in Sijtsma [18] leading to a CSM given by

$$
C_{j}=\vec{g}_{j}^{H} \vec{g}_{j}=\vec{w}_{j}^{H} \vec{w}_{j}
$$

where $\vec{w}_{j}$ is the steering vector for the unit power source. This allowed tracing a beamforming map by scanning grid points $\xi_{s}$ of interest so that

$$
b_{j s}=\vec{w}_{s} C_{j} \vec{w}_{s}^{H}=\vec{w}_{s}\left(\vec{w}_{j}^{H} \vec{w}_{j}\right) \vec{w}_{s}^{H}=\left\|\vec{w}_{s} \vec{w}_{j}^{H}\right\|^{2}
$$

i.e., the PSF map can be obtained by fixing the steering vector $\vec{w}_{j}$ at the source position while sweeping $\vec{w}_{s}$ over the image grid $\xi_{s}$. Regarding (9) for the modal steering vector, the duct PSF opens the possibility of allowing the proper visualization of effects due to the duct. For the USP fan rig geometry, a PSF simulation example can be seen in Fig. 2 for a source located at $r=a / 2=0.15 \mathrm{~m}, \theta=0$ and $z=-0.71 \mathrm{~m}$. The position of the focal plane ( $z$ distance) is the same as that of the fan plane. Results for six different frequencies are displayed. The USP array and its rig geometry are reviewed in Section IV.

Note that, due to the small number of cut-on modes at low frequencies, source location on the PSF map fails to exactly match the desired source position $\vec{j}$ under the beam wide main lobe. At $0.867 \mathrm{kHz}$ and $1.13 \mathrm{kHz}$, a clearly discernible source close to the wall becomes evident and for less than 12 cut-on modes. Strong beamforming side-lobes and large beam widths can also be seen. At higher frequencies, source discernibility increases followed by reduced side-lobe levels, thanks to a larger number of cut-on modes that enable better source location representation. Overall, these visualization results confirm code plausibility.

\section{ARray Signal Processing}

Beamforming relies directly on spectral estimation [see Section II, (1)]. The cross-spectral density from pressure signals from all the array microphones must be computed and one must always keep in mind the several practical limitations of spectral estimation methods. This vary according to signal characteristics leading to errors, such as spectral leakage, blurring (resolution), bias, and so on [19]-[21]. 


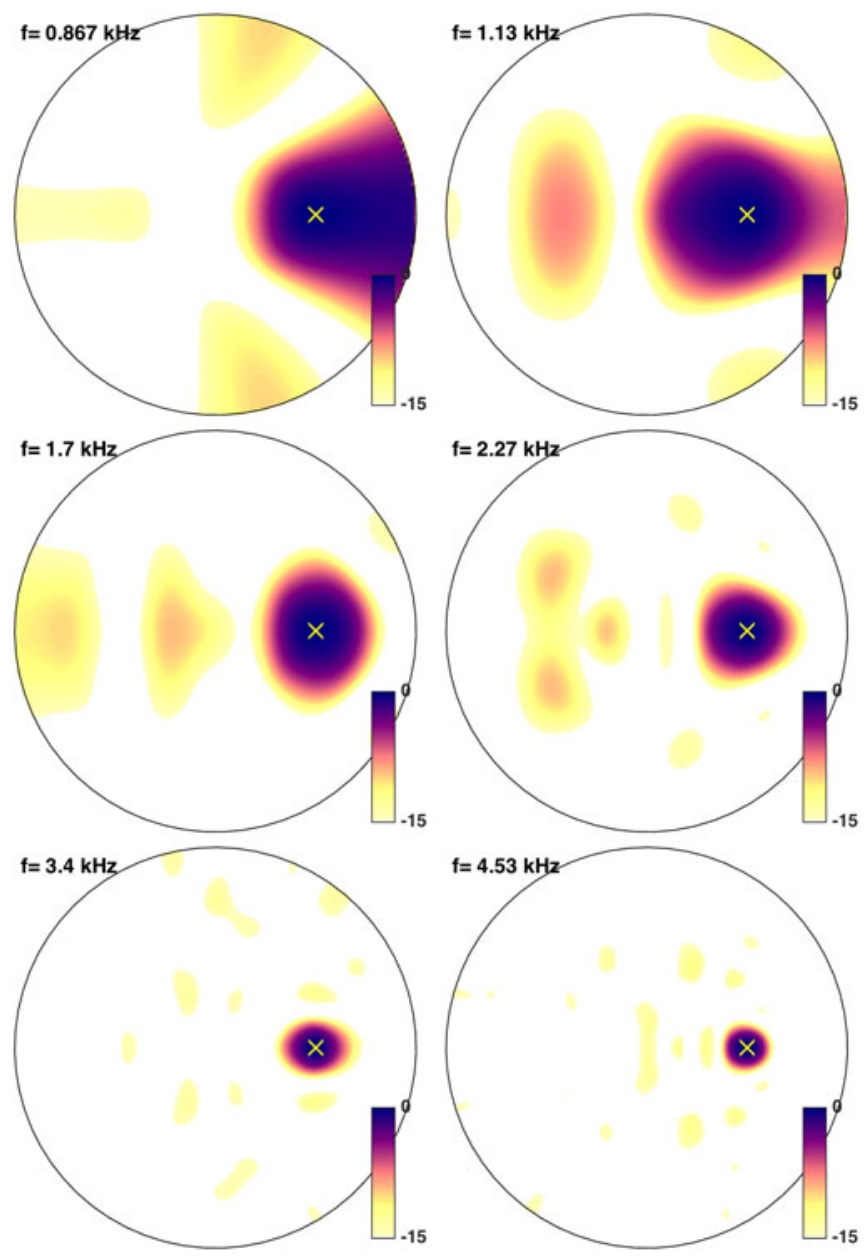

Fig. 2. PSF simulation at six frequencies for the USP fan rig/array configuration in Section IV at the fan plane using a unit dipole source placed at the half radius length $r=a / 2$ (marked with a small yellow cross). Neither Mach flow nor rotating effects are included. The color bar scale displays noise intensity in $\mathrm{dB}$ regarding unit pressure $p_{\text {ref }}=1 \mathrm{~Pa}$.

For the present experiments, microphone array data was synchronously acquired at $51.2 \mathrm{kHz}$ sampling rate for about 30 seconds of acquisition time. Because of this long data available, it is safe to apply the well known Welch spectral estimation method [20]. This consists of averaging Fourier transformed data over small (2048 sample long) data windows. The usual literature recommended $50 \%$ of overlap between signal segments [19] after applying the Hanning taper [3], [20] to each window to achieve a good trade-off between spectral resolution and reduced side-lobe levels.

\section{A. PSD Estimation Details}

Let two data records of two signals $\mathbf{x}$ and $\mathbf{y}$ of length comprising $N$ samples, be broken into window blocks $\mathbf{x}^{(k)}$ and $\mathbf{y}^{(k)}$ of size $L$. The cross spectrum estimate $\hat{S}_{x y}^{(k)}(f)$ for each $k$ block is given by

$$
\hat{S}_{x y}^{(k)}(f)=\frac{2 \cdot \mathcal{F}\left(\mathbf{x}^{(\mathbf{k})} \cdot \mathbf{w}\right) \cdot \operatorname{conj}\left(\mathcal{F}\left(\mathbf{y}^{(\mathbf{k})} \cdot \mathbf{w}\right)\right)}{f_{s} \cdot \sum w_{i}^{2}}
$$

where $\mathbf{w}=\left[w_{1} \ldots w_{i} \ldots w_{L}\right]^{T}$ is a suitable data taper window of length $L$ and $f_{s}$ is the sampling frequency. The normalization factor $\sum w_{i}^{2}$ comes from the fact that aeroacoustic noise signals [3] have continuous spectra deriving from their nature as regular stochastic processes, i.e., strictly devoid of sinusoidal tones. So, this factor is used to ensure that the power computed over time matches the one that can be obtained by integrating the continuous spectrum when $\mathbf{x}=\mathbf{y}$. This normalization is recommended when pure sinusoids are absent; see a detailed discussion in [22].

The symbol $\mathcal{F}\{\cdot\}$ stands for the discrete time Fourier transform computed at suitable frequencies $f$ between 0 and one half of the Nyquist frequency $\left(f_{s}\right)$. Factor 2 stems from using a single-sided representation (positive frequencies only) of the spectrum.

Finally, the cross-spectrum estimate is obtained by averaging over the block estimates (15):

$$
\hat{S}_{x y}(f)=\sum_{k=1}^{N / L} \frac{\hat{S}_{x y}^{(k)}(f)}{N / L}
$$

that are subsequently used to build-up the cross-spectrum matrix.

\section{B. The Cross Spectral Matrix (CSM)}

CSM estimation for fan noise analysis is not as direct as for most of free-field and wind-tunnel cases [3]. Instead, one must apply a sequence of signal processing techniques prior to beamforming.

First, we begin by removing strong fan tones (harmonics generated by the passage of the fan blades) from the data using a time domain algorithm that estimates and subtracts the corresponding sinusoids from the raw signal. Subsequently, the resulting data is time-resampled to an equivalent 512 samples per fan revolution, i.e., the final sampling frequency is dependent on fan speed. This is an important step because many noise sources generated by rotating machinery have a frequency that depend on shaft speed. The number of samples per fan revolution is optional, but here we use 512 points for convenience as it leads to more computationally efficient code. See [9], [14], [23], [24] for more details.

To construct the CSM all pairwise microphone combinations at a given frequency, $f_{0}$ are put together:

$$
\left[\mathbf{C}\left(f_{0}\right)\right]_{N x N}=\left[\begin{array}{cccc}
S_{11}\left(f_{0}\right) & S_{12}\left(f_{0}\right) & \cdots & S_{1 N}\left(f_{0}\right) \\
S_{21}\left(f_{0}\right) & S_{22}\left(f_{0}\right) & \cdots & S_{2 N}\left(f_{0}\right) \\
\vdots & \vdots & \ddots & \vdots \\
S_{N 1}\left(f_{0}\right) & S_{N 2}\left(f_{0}\right) & \cdots & S_{N N}\left(f_{0}\right)
\end{array}\right]
$$

where $S_{i j}\left(f_{0}\right)$ values come directly from the Welch spectral estimates (16).

As commonly adopted in aeroacoustic experiments [3], the main diagonal from the CSM was removed prior to beamforming calculations. This is highly recommended to avoid boundary layer noise when microphones are directly exposed to the flow. The presence of flow in front of microphones induces high levels 
of noise due to the turbulent boundary layer, and is unrelated to the noise sources of interest themselves.

\section{Virtual Rotating Microphones (VRM)}

To the best of our knowledge, the first approach to virtual rotating beamforming was proposed by Lowis [12] and employs a frequency domain signal representation. Recently, Pannert et al. published [13] experimental results for a rotating source under free-field wave propagation using the same approach Caldas et al. [14], [24] had previously examined for rotating arrays using time domain and frequency domain approaches that directly compensate for rotation using modal steering vectors.

Since array data are already available in the frequency domain, the technique consists of applying a spatial Fourier transformation to a ring of microphones to obtain "mode domain" data representation. Once in this space, each mode order " $\mathrm{m}$ " can be frequency-shifted to compensate for the motion effect of the sound source. This procedure is followed by inverse spatial Fourier transformation leading to mode compensated data. Lowis and Pannert demonstrated that this is equivalent to would-be rotating microphones at the same speed as the fan.

Mathematically, owing to in-duct sound field periodicity in the $\theta$ direction, the measured pressure $p$ at a ring of $N_{c}$ equally spaced microphones can be written as a discrete Fourier series as

$$
\begin{array}{r}
p_{m}(z, a, \omega)=\frac{1}{N_{c}} \sum_{i=1}^{N_{c}} p\left(z, a, \theta_{i}, \omega\right) e^{-j m \theta_{i}}, \\
\left(\frac{-\left|1-N_{c}\right|}{2} \leq m \leq \frac{N_{c}}{2}\right)
\end{array}
$$

where $\theta_{i}$ and $z$ are the $i$ th microphone azimuthal position and distance to a reference plane, respectively and $p_{m}$ is the pressure amplitude of the $m$ th spinning mode.

Once in this "mode domain", the sound pressure of the virtual rotating microphones is obtained by the inverse spatial Fourier transform of the frequency shifted spinning modes $p_{m}(z, a, \omega+m \Omega)$ as

$$
p_{\Omega}\left(z, a, \theta_{i}, \omega\right)=\sum_{m=-m-}^{m+} p_{m}(z, a, \omega+m \Omega) e^{+j m \theta_{i}}
$$

where $m+$ and $m$ - are the same as in (18), $\Omega$ is the shaft speed and $p_{\Omega}$ already represents the sound pressure at the $i$ th microphone in the rotating reference frame. The term $\omega+m \Omega$ selects different frequency terms in $p_{m}$ depending on the order of the spinning mode and on the shaft speed. This technique is roughly similar to a signal modulation process [19].

\section{EXPERIMENTAL SETUP}

The experimental facility is composed of a long-duct lowspeed fan rig test bed at the São Carlos Engineering School of the University of São Paulo (USP) in Brazil, showed in Fig. 3. Its long duct configuration is similar to that of DLR's low speed fan rig [25] but differs from other rigs that operate inside anechoic chambers [11], [26].

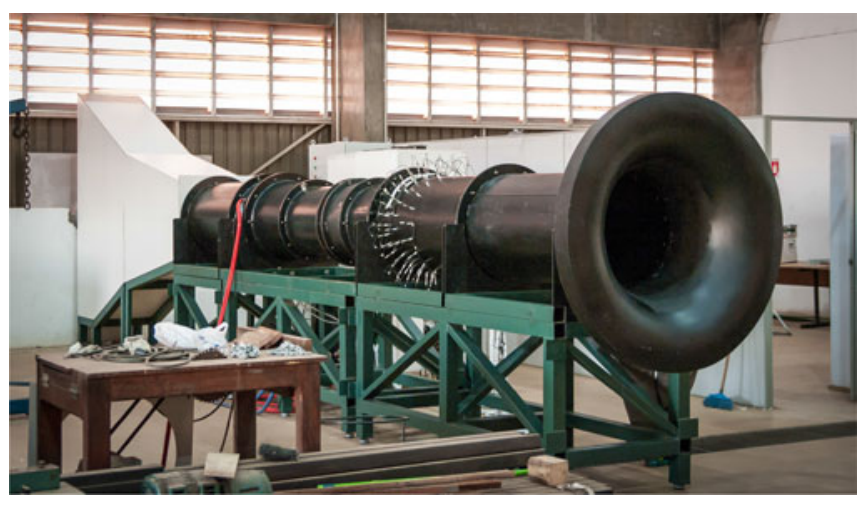

Fig. 3. Photographic illustration of the USP fan rig. The bell-mouth inlet is portrayed with its 77-microphone array (upstream from the rotor). Orange cables connecting the duct are the motor power supply. Downstream, the rig has a white anechoic termination.

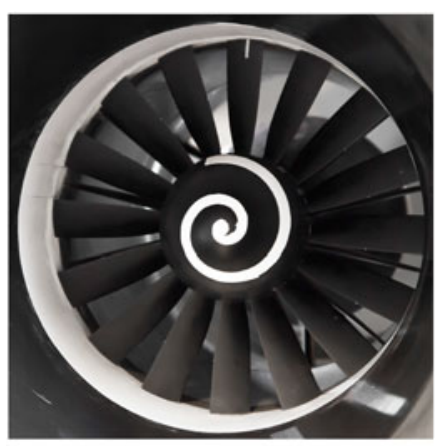

(a)

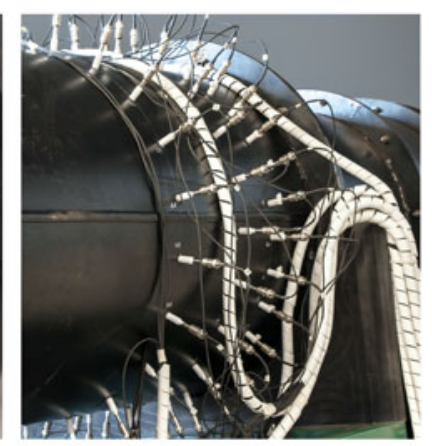

(b)
Fig. 4. Look-up at the 16-bladed rotor fan and the wall-flush mounted 77microphone array. (a) 16-bladed fan. (b) The 3-rings 77-microphones array.

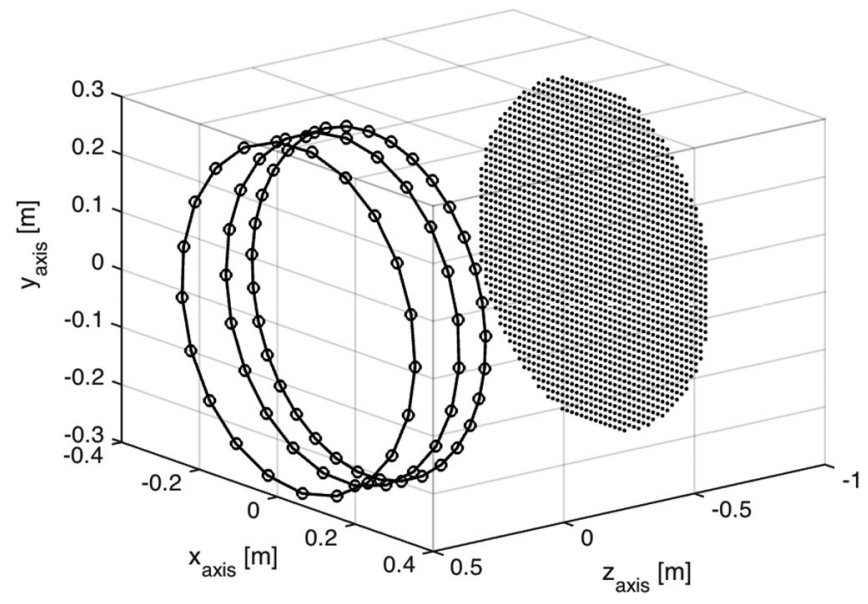

Fig. 5. 77-microphone array (circles) and the focal plane represented by the black dots placed at the fan distance.

The array comprises 77-microphones flush-mounted microphones (GRAS 40PH-S2 $\phi 7 \mathrm{~mm}$, bandwidth of 20 to $20 \mathrm{kHz}$ and $50 \mathrm{mV} / \mathrm{Pa}$ average sensitivity) as portrayed in Fig. 4(a) and consists of three rings 0.10 and $0.17 \mathrm{~m}$ apart (Figs. 4(b) and 5). The ring closer to the fan lies at $0.86 \mathrm{~m}$ from it and has 

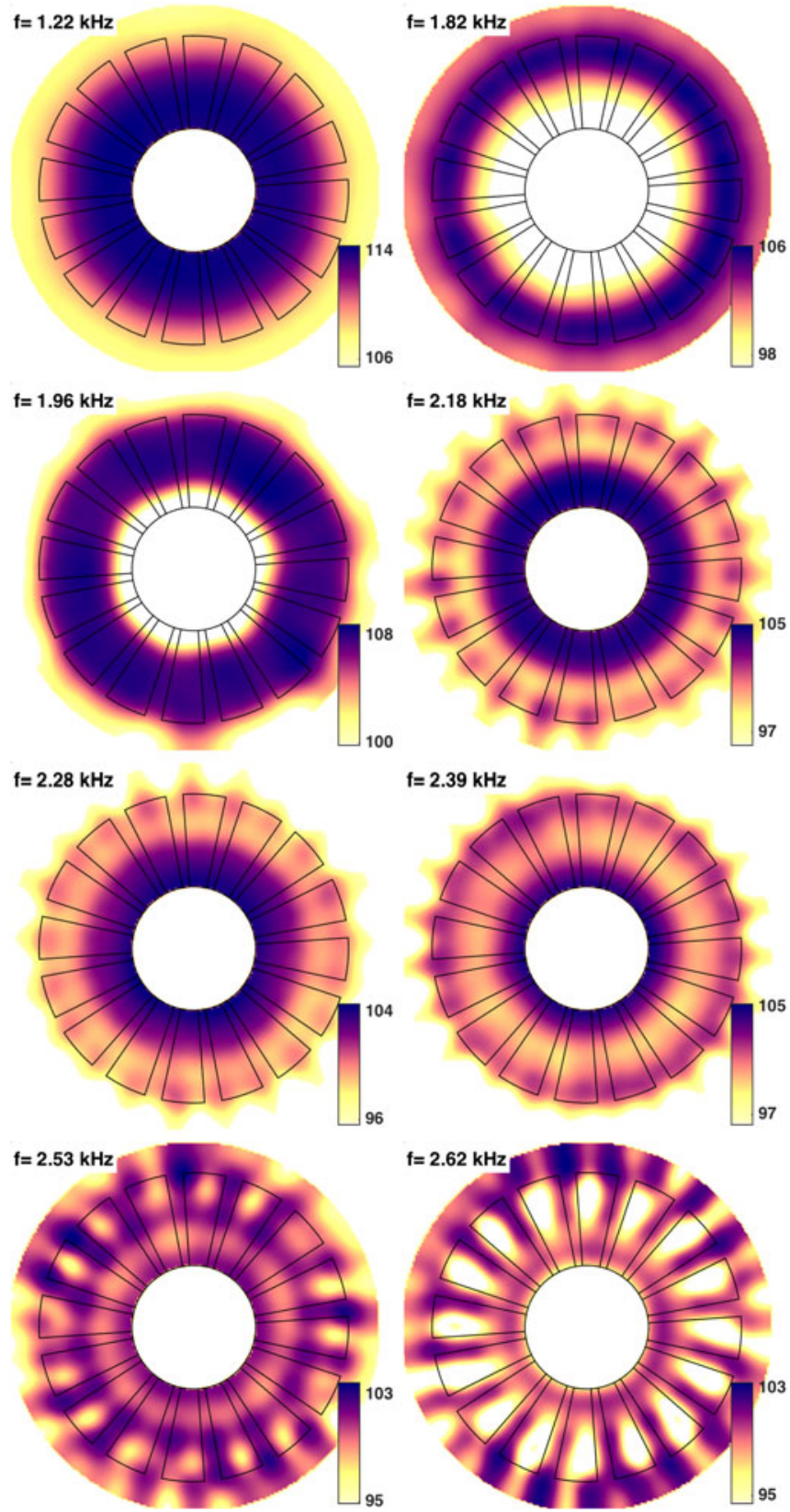

Fig. 6. Conventional rotating beamforming maps for 8 different frequencies between $1 \mathrm{kHz}$ to $3 \mathrm{kHz}$ (labeled at the left-top of each plot) at a $4250 \mathrm{rpm}$ rotating fan speed and Mach flow of 0.091. The focal plane was chosen on top of the fan. The color bar scale displays noise intensity in $\mathrm{dB}$ referred to $p_{\text {ref }}=20 \mu \mathrm{Pa}$

33 uniformly distributed microphones, whereas the other rings have 23 and 21 microphones, respectively. A PXI National Instruments system with 5 PXI-4496 boards is used for data acquisition. Angular motor position is also simultaneously acquired.

\section{EXPERIMENTAL RESULTS}

Figs. 6 and 7 portray 16 acoustic maps at different frequencies at a fan rotation speed of 4,250 rpm and with the focal plane placed on top of the fan. Black lines approximately denote the position of each fan blade. The center circle denotes the fan spin-
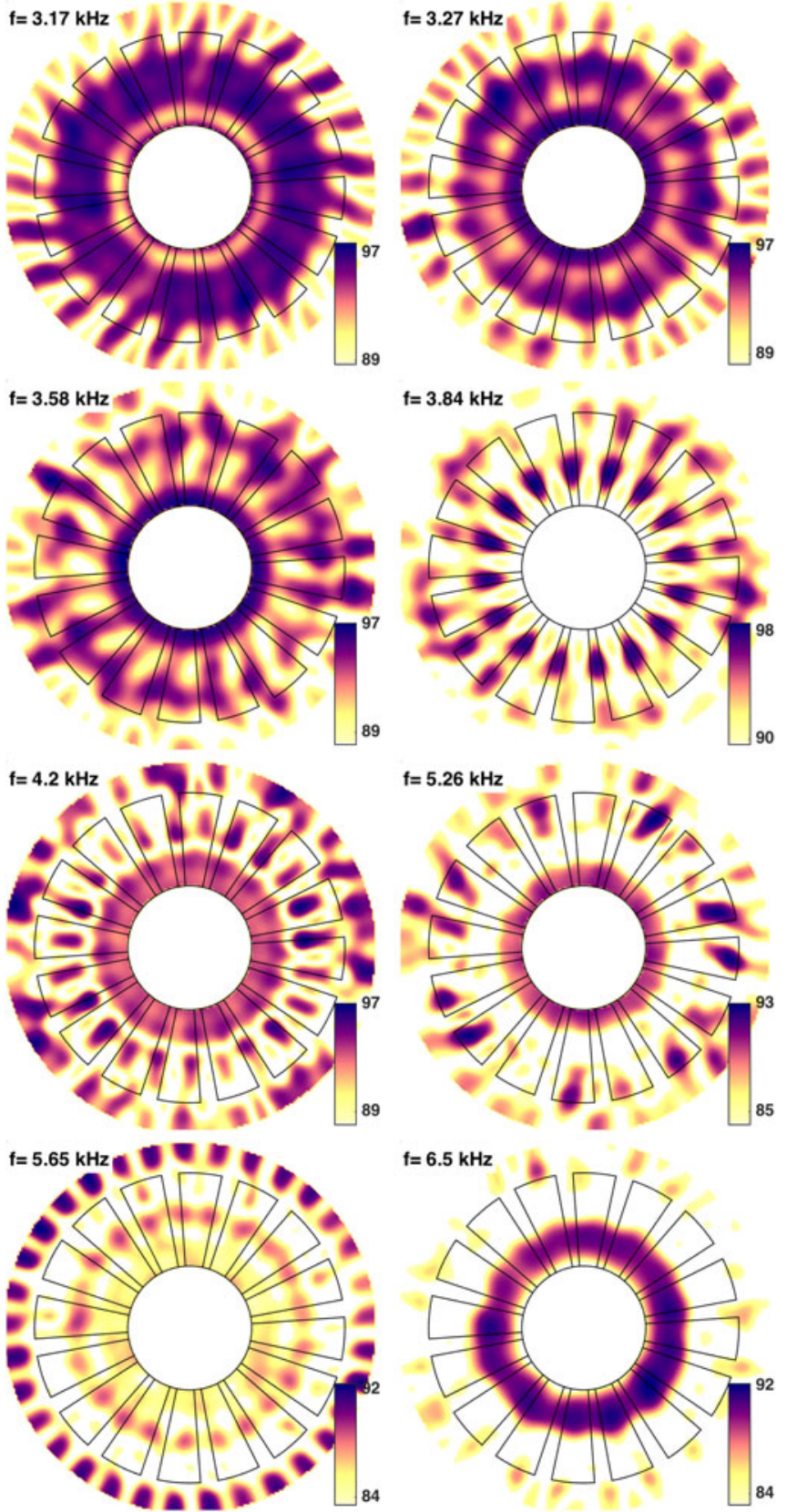

Fig. 7. Conventional rotating Beamforming maps for 8 different frequencies between $3 \mathrm{kHz}$ to $7 \mathrm{kHz}$ (labeled at the left-top of each plot) at a $4250 \mathrm{rpm}$ rotating fan speed and Mach flow of 0.091. The focal plane rests on top of the fan. The color bar scale displays noise intensity in $\mathrm{dB}$ referred to $p_{\text {ref }}=20 \mu \mathrm{Pa}$.

ner. The noise coming from this part is hidden for convenience, since it is not a real source of noise and only a by-product of reflections that may happen. The color bar scale displays noise intensity in $\mathrm{dB}$ referred to $p_{\text {ref }}=20 \mu \mathrm{Pa}$. As detailed in Section IV, the duct diameter in the array stage is $0.6 \mathrm{~m}$ and equals $0.5 \mathrm{~m}$ at the fan stage. However, for our acoustic modeling we consider only a constant $0.6 \mathrm{~m}$ duct section. Indeed, this is the reason why the plotted blade tips do not touch the duct wall in Figs. 6 and 7.

At low frequencies in Fig. 6, the top maps for $1.22 \mathrm{kHz}$ to $2 \mathrm{kHz}$, do not discriminate between the noise sources due to each 
single blade because of the large beam-width associated with the small number of available cut-on modes in conformity to the PSF maps of Fig. 2. In other words, the noise sources look more "smeared" around the blades. However, beyond about $2.16 \mathrm{kHz}$ discrete noise sources at the blade tips show up clearly and smeared noise sources appear at the blade roots (which connect the blades the spinner). This is also verified for $2.28 \mathrm{kHz}$ and $2.36 \mathrm{kHz}$.

At $2.53 \mathrm{kHz}$ and $2.62 \mathrm{kHz}$, the contribution from each blade becomes even more evident, as the pattern of 16 sources is clearly distinguishable. Artifacts close to the duct wall also start to be evident. It is worth mentioning that these artifacts are indeed not sources of noise, but represent spurious effects appearing due to array geometry limitations in accord with the same phenomenon present in the PSF plots of Fig. 2.

For the second set of plots (see Fig. 7), the top plots show some discernible discrete noise sources in the region of the fan blades together with a smeared background. Artifacts are also evident close to the duct wall. At $3.84 \mathrm{kHz}$, a clear noise signature from each single blade is observed. At $4.2 \mathrm{kHz}$ and $5.26 \mathrm{kHz}$, the smeared noise from the blade roots are again observed with some artifacts at the fan area.

Finally, for the highest frequencies, $5.65 \mathrm{kHz}$ and $6.5 \mathrm{kHz}$, many artifacts close to the duct wall are observed together with some mid blade noise. At the last frequency, smeared blade root sources are observed.

\section{CONCLUSION}

Beamforming has been frequently used for locating noise sources in aeroacoustics [3], [4], [27]. However, recent tests have been performed in unconventional environments and conditions, such as within a duct under rotating noise sources [9], [11], [12], [23], [24] where we could show it to be a feasible tool for spotting noise sources.

Differently from Dougherty et al. [9], the present rotating array technique is completely implemented in the frequency domain. All this following Pannert's [13] free-field suggestions but applied here to ducted sources in an actual experimental setup for the first time. The present results are preliminary but match those observed in the references under different techniques/setups.

\section{APPENDIX A \\ MODE NORMALIZATION $N_{m, n}^{2}$}

The mode normalization term $N_{m, n}^{2}$ in (9) must satisfy the energy conservation condition over a cross sectional surface $\mathbf{S}$, which, in this case, corresponds to a circular disk with radius $a$

$$
S^{-1} \int_{\mathbf{S}}|\varphi(\vec{r})|^{2} d \mathbf{S}=1
$$

whose evaluation leads to

$$
N_{m, n}^{2}=\left(1-\frac{m^{2}}{\left(k_{m, n} a\right)^{2}}\right)\left|J_{m}\left(k_{m, n} a\right)\right|^{2}
$$

\section{ACKNOWLEDGMENT}

The USP fan rig test facility was designed and assembled by technicians and engineers of USP-São Carlos and Escola Politécnica Engineering Schools with the cooperation from EMBRAER's engineering team, inside the AERONAVE SILENCIOSA Project. The authors would like to thank all those involved in this project, including NASA Glenn Research Center's staff, specially Dr. D. Sutliff, whose support and technical insight proved very helpful. Special thanks go to B. Rocamora, M.Sc. student, supported by the same project and who helped run all the experiments.

\section{REFERENCES}

[1] Y. Guo and M. Joshi, "Noise characteristics of aircraft high lift systems," J. Aircr, vol. 41, no. 7, pp. 1247-1256, 2003.

[2] C. C. Pagani, D. S. Souza, and M. A. F. Medeiros, "Slat noise: Aeroacoustic beamforming in closed-section wind tunnel with numerical comparison," AIAA J., vol. 54, no. 7, pp. 2100-2115, 2016.

[3] T. J. Mueller, Aeroacoustic Measurements. Berlin, Germany: Springer 2001.

[4] W. Dobrzynski, "Almost 40 years of airframe noise research: What did we achieve," J. Aircr., vol. 47, no. 2, pp. 353-367, Mar./Apr. 2010.

[5] T. F. Brooks and W. M. Humphreys, "Effect of directional array size on the measurement of airframe noise components," in Proc. 5th AIAA/CEAS Aeroacoust. Conf., 1999.

[6] Y. Guo, K. Yamamoto, and R. Stoker, "Component-based empirical model for high-lift system noise prediction," J. Aircr., vol. 40, no. 5, pp. 914-922, 2003.

[7] R. Merino-Martínez, M. Snellen, and D. G. Simons, "Functional beamforming applied to imaging of flyover noise on landing aircraft," J. Aircr., vol. 53, no. 6, pp. 1830-1843, 2016.

[8] R. P. Dougherty and J. M. Mendonza, "Nacelle in-duct beamforming using modal steering vectors," in Proc. 14th AIAA/CEAS Aeroacoust. Conf./29th AIAA Aeroacoust. Conf., 2008, Paper 2812. [Online] Available: http://dx.doi.org/10.2514/6.2008-2812

[9] R. P. Dougherty and B. E. Walker, "Virtual rotating microphone imaging of broadband fan noise," in Proc. 15th AIAA/CEAS Aeroacoust. Conf./30th AIAA Aeroacoust. Conf., 2009, Paper 3121. [Online]. Available: http://dx.doi.org/10.2514/6.2009-3121

[10] R. P. Dougherty, B. E. Walker, and D. L. Sutliff, "Locating and quantifying broadband fan sources using in-duct microphones," in Proc. 16th AIAA/CEAS Aeroacoust. Conf., 2010, Paper 3736. [Online]. Available: http://dx.doi.org/10.2514/6.2010-3736

[11] P. Sijtsma, "Feasibility of in-duct beamforming," in Proc. 13th AIAA/CEAS Aeroacoust. Conf./28th AIAA Aeroacoust. Conf., 2007, Paper 3696.

[12] C. R. Lowis, "In-duct measurement techniques for the characterisation of broadband aeroengine noise," Ph.D. dissertation, Univ. Southampton, Southampton, U.K., 2007.

[13] W. Pannert and C. Maier, "Rotating beamforming—Motion-compensation in the frequency domain and application of high-resolution beamforming," J. Sound Vib., vol. 333, no. 7, pp. 1899-1912, 2014.

[14] L. C. Caldas, P. C. Greco, C. C. Pagani, and L. A. Baccala, "Comparison of different techniques for rotating beamforming at the University of São Paulo fan rig test facility," in Proc. 6th Berlin Beamforming Conf., no. BeBeC-2016-D14, 2016.

[15] C. L. Beattie, "Table of first 700 zeros of Bessel functions- $J_{l}(x)$ and $J_{l}^{\prime}(x)$, , Bell Syst. Tech. J., vol. 37, no. 3, pp. 689-697, May 1958.

[16] S. Rienstra and A. Hirschberg, An Introduction to Acoustics. Eindhoven, The Netherlands: Eindhoven Univ. Technol., 2014.

[17] L. C. Caldas, R. G. Cuenca, R. Q. Lauterjung, and L. A. Baccala, "In-duct beamforming noise source estimation and mode detection at the University of São Paulo fan rig," in Proc. 21 st AIAA/CEAS Aeroacoust. Conf., 2015, Paper 2233. [Online]. Available: http://dx.doi.org/10.2514/6.2015-2233

[18] P. Sijtsma, "CLEAN based on spatial source coherence," in Proc. 13th AIAA/CEAS Aeroacoust. Conf./28th AIAA Aeroacoust. Conf., 2007, Paper 3436.

[19] D. B. Percival and A. T. Walden, Spectral Analysis for Physical Applications. Cambridge, U.K.: Cambridge Univ. Press, 1993.

[20] S. M. Kay, Modern Spectral Estimation: Theory \& Application Englewood Cliffs, NJ, USA: Prentice-Hall, 1987. 
[21] P. Stoica and R. L. Moses, Introduction to Spectral Analysis. Englewood Cliffs, NJ, USA: Prentice-Hall, 1997.

[22] M. Trbs and G. Heinzel, "Improved spectrum estimation from digitized time series on a logarithmic frequency axis," Measurement, vol. 39, no. 2, pp. 120-129, 2006. [Online]. Available: dx.doi.org/10.1016/j. measurement.2005.10.010

[23] L. C. Caldas, "In-duct beamforming and mode detection using a circular microphone array for the characterisation of broadband aeroengine fan noise," M.S. thesis, Escola politécnica da Universidade de São Paulo, São Paulo, Brazil, 2016.

[24] L. C. Caldas, G. Herold, P. C. Greco, and L. A. Baccala, "In-duct rotating beamforming and mode detection of fan noise sources," in Proc. AIAA 22st AIAA/CEAS Aeroacoust. Conf., 2016, Paper 3034. [Online]. Available: http://dx.doi.org/10.2514/6.2016-3034

[25] L. Enghardt, A. Moreau, and P. Kausche, "Active control of fan tones by means of trailing edge blowing," in Proc. 21st AIAA/CEAS Aeroacoust. Conf., 2015, Paper 2828. [Online]. Available: http://dx.doi.org/ $10.2514 / 6.2015-2828$

[26] R. A. Loew, J. T. Lauer, J. MCAllister, and D. L. Sutliff, "The advanced noise control fan," in Proc. 25th AIAA Aerodyn. Meas. Technol. Ground Test. Conf., no. NASA/TM-2006-214368, Also AIAA-2006-3150, 2006.

[27] P. Sijtsma, "Phased array beamforming applied to wind tunnel and fly-over tests," SAE Int., Warrendale, PA, USA, Tech. Rep. 2010-36-0514, 2010.

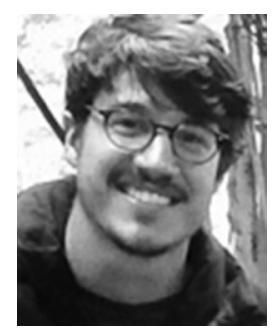

Luciano C. Caldas (M'17) received the Bachelor's and M.Sc. degrees in electrical engineering from the University of São Paulo, campus of São Carlos and São Paulo, respectively, Brazil, in 2012 and 2016, respectively. Recently, he was involved in a project aiming the construction of a fan rig test facility for fan noise characterization. He developed signal processing codes based on microphone array data for in-duct acoustic wave propagation analysis, as well rotating beamforming and mode detection algorithms for noise source localization. His research interests include multivariate signal processing, acoustics, digital and analog electronics, optimization, and embedded systems.

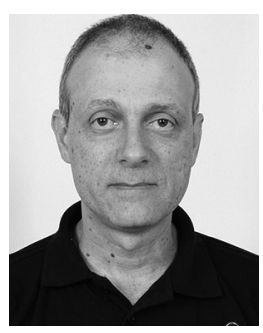

Paulo C. Greco Jr. received the B.S. degree in mechanical engineering from the University of São Paulo, São Carlos, Brazil, in 1987, and the M.S. and $\mathrm{Ph} . \mathrm{D}$. degrees in aerospace engineering, both from the University of Kansas, Lawrence, KS, USA, in 1993 and 1996, respectively. He is currrently an Associate Professor at the University of São Paulo. $\mathrm{He}$ has experience in aerospace engineering, focusing on aeroelasticity, acting on the following subjects: Computational aerodynamics and aeroacoustics.

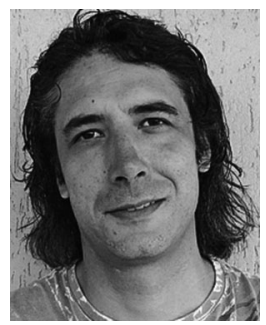

Carlos C. Pagani received the B.S. degree in physics from Federal University of São Carlos, São Carlos, Brazil, in 2007, and the M.S. degree in mechanical engineering and the Ph.D. degree in aeronautical engineering, both from the University of São Paulo, São Paulo, Brazil, in 2009 and 2014, respectively. $\mathrm{He}$ is currently an Assistant Professor at São Paulo State University, campus of São João da Boa Vista, São Paulo, Brazil. His main research fields are aeroacoustics and structural dynamics.

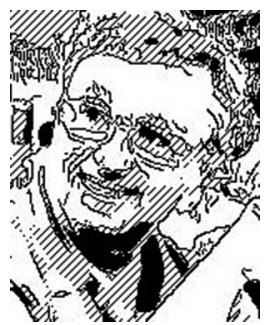

Luiz A. Baccalá (M'93) majored in electrical engineering and physics, in 1984, from the University of São Paulo, São Paulo, Brazil, where he received the M.Sc. degree in 1991, studying the time-series evolution of bacterial resistance to antibiotics in a nosocomial environment. Since then, he has been involved in statistical signal processing and analysis and received the Ph.D. degree from the University of Pennsylvania, Philadelphia, PA, USA, in 1995 , working on new statistical methods of communication channel identification and equalization. Since his return to his alma mater, he has taught courses in applied stochastic processes and advanced graduate-level statistical signal-processing courses including wavelet analysis and spectral estimation. His current research interests include investigation of multivariate time-series methods for neural connectivity inference and for problems of inverse source determination using arrays of sensors that include functional magnetic resonance imaging and multielectrode electroencephalogram processing. 